\title{
La Educación Física argentina en la actualidad: normativas, lineamientos curriculares, formación docente y desempeño laboral
}

\author{
Argentine Physical Education today: regulations, curricular guidelines, teacher training and job performance \\ A Educação Física Argentina hoje: normas, diretrizes curriculares, formação de professores e desempenho \\ profissional
}

\author{
Alejo Levoratti ${ }^{1}$; Pablo Kopelovich ${ }^{2}$ \\ Universidad Nacional de La Plata, UNLP, La Plata, Argentina
}

\begin{abstract}
RESUMEN
Presentamos un panorama general de la situación actual de la Educación Física escolar en la República Argentina. Para ello, abordamos distintas problemáticas como su normativa, la formación docente (de grado y posgrado), el desempeño profesional, su orientación predominante, la prohibición de la separación de los grupos por sexo, entre otras cosas. A nivel jurisdiccional, priorizamos el caso de la provincia de Buenos Aires. Nos basamos en distintas normativas nacionales y provinciales, informes de entidades estatales, ponencias, tesis y artículos académicos. Incluimos, al final del texto, una serie de desafíos que entendemos que es necesario afrontar para continuar mejorando el estado de la disciplina en cuestión en el país que nos compete.

Palabras clave: Educación Física. Normativa. Formación Docente. Desempeño Profesional.
\end{abstract}

\begin{abstract}
We present an overview of the current situation of school physical education in the Argentine Republic. To this end, we address different problems such as their regulations, teacher training (bachelor and postgraduate), professional performance, their predominant orientation, the prohibition of the separation of groups by sex, among other things. At the jurisdictional level, we prioritize the case of the Province of Buenos Aires. We rely on various national and provincial regulations, reports from state entities, lectures, theses and academic articles. We include, at the end of the text, a number of challenges that we understand need to be faced in order to continue improving the state of the discipline in question in the country under our jurisdiction.
\end{abstract}

Keywords: Physical Education. Regulations. Teacher Training. Professional Performance.

\section{RESUMO}

Apresentamos uma visão geral da situação actual da Educação Física escolar na Argentina. Para isto, abordamos diferentes questões tais como os seus regulamentos, formação de professores (graduação e pós-graduação), desempenho profissional, a sua orientação predominante, a proibição da separação de grupos por sexo, entre outras coisas. Em nível jurisdicional, damos prioridade ao caso da província de Buenos Aires. Baseamos-nos em distintos regulamentos nacionais e provinciais, relatórios de agências governamentais, trabalhos, teses e artigos acadêmicos. Incluimos, no final do texto, alguns desafios que consideramos necessário enfrentar a fim de continuar a melhorar o estado da disciplina em questão no país em questão.

Palavras-chave: Educação Física. Regulamentos. Formação de Professores. Desempenho Profissional.

\footnotetext{
1 Investigador del Consejo Nacional de Investigaciones Científicas y Técnicas (CONICET) y Docente investigador de la UNLP. E-mail: levoratti@gmail.com. ORCID: https://orcid.org/0000-0003-2749-0321.

2 Becario doctoral del CONICET y Profesor de la UNLP, Departamento Educación Física. E-mail: kopelovichp@gmail.com. ORCID: https://orcid.org/0000-0003-3708-9881.
} 


\section{INTRODUCCIÓN}

En el presente artículo nos proponemos dar un panorama general sobre la situación de la Educación Física en Argentina en la actualidad, haciendo especial hincapié en la provincia de Buenos Aires. Nos encargamos de dar cuenta de las características que adquiere la formación docente en el país, así como el desempeño profesional y la orientación impresa a esta asignatura.

La Argentina es un país soberano de América del Sur, ubicado en el extremo sur y sudeste de dicho continente. Limita al norte con Bolivia y Paraguay, al nordeste con Brasil, al este con Uruguay y el océano Atlántico, al oeste con Chile y, siempre en su sector americano, al sur con Chile y las aguas atlánticas del pasaje de Drake. Adopta la forma de gobierno republicana, democrática, representativa y federal. Su actual presidente es Alberto Fernández, del partido Justicialista.

Es un país bicontinental con una superficie de $2.780 .400 \mathrm{~km}^{2}$, el hispanohablante más extenso del planeta, siendo el segundo más grande de América Latina y octavo en el mundo, si se considera solo la superficie continental sujeta a soberanía efectiva. Su plataforma continental, reconocida por la ONU en 2016, alcanza los $6.581 .500 \mathrm{~km}^{2}$ (ARGENTINA, 2022), convirtiéndose en una de las más grandes del mundo. Su capital es la Ciudad Autónoma de Buenos Aires y está compuesto por 23 provincias, que cuentan con autonomía plena. Según el último censo de 2019, su población es 44.938 .712 habitantes. El sistema educativo argentino lo integran instituciones tanto de gestión pública como privadas, estando descentralizado hacia sus jurisdicciones provinciales. De acuerdo a datos del Sistema de Información de Tendencias Educativas en América Latina (SITEAL) en 2016 el 99\% de los niños y niñas de entre 6 y 11 años estaba escolarizado, mientras que el 88,5\% de los jóvenes de entre 15 a 17 años asistía a establecimientos de nivel secundário (UNESCO, 2016). Asimismo, el sistema educativo de la provincia de Buenos Aires es el tercero más grande de América Latina.

Con este cuadro de situación estructuramos el artículo en tres apartados. En el primero, aludimos a la Educación Física a partir de su normativa, dando cuenta de legislación tanto nacional como jurisdiccional. En el segundo, presentamos cómo fue y es la formación docente en la materia en el país, describiendo las diferentes modalidades existentes. Finalmente, nos encargamos de una serie de cuestiones no incluidas en las secciones anteriores, pero que estimamos dignas de mención, como son la deportivización de la disciplina, los ambitos de desempeño profesional, las competencias deportivas escolares, y la división de los grupos por sexo para las clases en ambitos escolares.

Para cumplir con ello nos adentramos en los marcos normativos, diseños curriculares y una serie de producciones que analizaron diferentes problemáticas vinculadas a la educación física, su inscripción en el sistema educativo y sus desafíos.

\section{La Educación Física en la normativa}

La Educación Física en Argentina está legislada por normativa tanto nacional como jurisdiccional (que incluye a las provincias y a la Ciudad Autónoma de Buenos Aires).

Así, dentro del Ministerio de Educación Nacional, se halla la Secretaría de Educación, que a su vez contiene la Subsecretaría de Educación Social y Cultural. Ésta, incluye el Área Nacional de Educación Física, deporte y salud, que “[...] se propone generar lineamientos y estrategias para la formación de ciudadanas y ciudadanos críticos y conscientes de sus derechos y responsabilidades, especialmente en materia de alimentación, actividad física, salud integral y calidad de vida" (ARGENTINA, 2021, n.p.). Además, busca acompañar y 
fortalecer la tarea desarrollada por las áreas de Educación Física jurisdiccionales a partir de una construcción federal y afianzar su gestión en el Sistema Educativo, a la vez que enriquecer las trayectorias educativas de niñas, niños y jóvenes a partir de acciones que permitan su enseñanza desde una perspectiva de derechos, con inclusión y sin estereotipos de género.

Con el objetivo de brindar las dimensiones necesarias para situar la enseñanza de la disciplina en el Sistema Educativo se aprobó, por Resolución n. 272 en 2019 de la Secretaría de Gestión Educativa, un documento marco de organización de la Educación Física nacional. Este propone un enfoque actualizado e innovador, para garantizar el derecho a la "educación corporal" a través de trayectorias escolares continuas, completas y significativas. En dicha resolución, se considera a la Educación Inclusiva como punto de partida para pensar la Educación Física escolar, estableciéndose que "Asume la responsabilidad de estudiar la cultura corporal de movimiento, teniendo como finalidad potenciar a los estudiantes para intervenir de forma autónoma, crítica y creativa en esa dimensión de la vida social" (ARGENTINA, 2019, p. 11). En ese marco, se estima, también, que debe pedagogizar las prácticas corporales sistematizadas y las representaciones sociales vinculadas a las mismas, de tal manera que proporcione al sujeto el acceso a una dimensión de conocimiento que no sería posible de alcanzar de otro modo.

En este contexto, las prácticas corporales expresadas como contenidos de la Educación Física en los actuales diseños curriculares tanto nacionales como jurisdiccionales son prácticas deportivas, gimnásticas, en el ambiente natural, ludomotrices, motrices expresivas, acuáticas y tradicionales, autóctonas y de otras culturas.

Entonces, a nivel nacional, los Núcleos de Aprendizajes Prioritarios (NAP), entendidos como conjunto de saberes centrales, relevantes y significativos, constituyen una base común para la enseñanza en todo el país. Estos se organizan por nivel: inicial, primario y secundario. En los últimos dos casos, se presentan por áreas y por ciclos, siendo los vigentes los del año 2004 (ARGENTINA, 2004a; 2004b) y 2011 (ARGENTINA, 2011). Con la vigente Ley Nacional de Educación (ARGENTINA, 2006a), los NAP reemplazaron a los Contenidos Básicos Comunes (CBC), propuestos por la Ley Federal de Educación (ARGENTINA, 1993), diferenciándose de estos por ser grandes orientaciones en vez de contenidos.

Así, en lo que respecta a los NAPs de Educación Física para nivel primario, se centran en conceptos como participación, inclusión, resolución de problemas, la creatividad, la percepción y la imaginación en relación a juegos (de cooperación-oposición, y tradicionales o regionales), actividades en el ambiente natural y habilidades motrices. Todo ello se produce en la búsqueda del cuidado de sí mismo, de los otros y del ambiente. Para el nivel secundario, estos núcleos plantean que las finalidades de la Educación Física son la disponibilidad corporal de sí mismo en interacción con el ambiente y con los otros, y la apropiación crítica de la cultura corporal y motriz. Para ese nivel, se incorporan también las prácticas deportivas, en la pretensión de que sean inclusivas, saludables y equitativas, respetuosas y que consideren la interacción entre los géneros y la atención a la diversidad.

Dicho esto, es necesario aclarar que la Educación Física es una asignatura obligatoria, a nivel nacional, a lo largo de 13 años (último año de nivel inicial, 6 años de escuela primaria y 6 años de escuela secundaria).

A nivel jurisdiccional, los diseños curriculares también se organizan por niveles, siendo los vigentes para la provincia de Buenos Aires los de 2018 (ARGENTINA, 2018a; 2018b). Allí, la Educación Física es concebida como práctica social que se manifiesta en diferentes actividades motrices propias de la cultura y como disciplina pedagógica en el ámbito educativo. De esta manera, interviene intencional y sistemáticamente en la constitución de la corporeidad y motricidad de los sujetos, en la apropiación de bienes 
culturales específicos, como las prácticas gimnásticas, atléticas, acuáticas, de vinculación con el ambiente natural, ludomotrices, motrices expresivas y deportivas. En este sentido, "[...] la Educación Física contribuye a la formación integral de los sujetos, considerando a los estudiantes en su complejidad humana y en sus posibilidades de comprender y transformar su realidad individual y social" (BUENOS AIRES, 2018a, p. 372).

A su vez, la provincia de Buenos Aires, desde el año 2010 ofrece, para el Ciclo Superior de la Escuela Secundaria una serie de orientaciones: Ciencias Sociales, Ciencias Naturales, Economía y Administración, Arte, Comunicación, Lenguas Extranjeras y Educación Física. En lo que respecta a esta última,

\begin{abstract}
La Escuela Secundaria Orientada en Educación Física comprende un conjunto de conocimientos y experiencias que permiten a los jóvenes profundizar saberes corporales y motrices en ámbitos variados, para la conquista de su disponibilidad corporal y motriz y para tener acceso a la cultura corporal de modo reflexivo, crítico y fundamentado. Fortalece el vínculo con los otros y las formas de actuar en el desarrollo de proyectos comunitarios referidos a prácticas corporales y motrices. Tiene materias específicas donde se realizan prácticas deportivas, acuáticas, gimnásticas, salidas de campamento y otras experiencias en ámbitos naturales (BUENOS AIRES, 2010, p. 7).
\end{abstract}

Se lleva a cabo, al igual que las otras orientaciones ofertadas, en el Ciclo Superior de la Enseñanza Secundaria; es decir, en 4to, 5to y 6to año. Los/as estudiantes reciben una formación común y una formación orientada. La Educación Física comprende un conjunto de conocimientos y experiencias que les permiten: ampliar y profundizar saberes corporales y motrices en ámbitos variados para obtener una plena disponibilidad corporal y motriz, de modo reflexivo, crítico y fundamentado; fortalecer el vínculo con los otros e integrarse activamente en los contextos socioculturales en los que habitan; conocer el campo de la cultura corporal y las formas de actuar en el desarrollo de proyectos de prácticas corporales y motrices para distintos grupos de la comunidad (BUENOS AIRES, 2010).

Las materias específicas para cada año, en cuarto año son: Educación Física y Corporalidad y Prácticas Deportivas y Atléticas; en quinto año tienen: Educación Física y Cultura, Prácticas Gimnásticas y Expresivas 1, Prácticas Deportivas y Acuáticas y Corporeidad y Deportes en Ámbitos Naturales. Por último, en el sexto año las cuatro materias son: Educación Física y Comunidad, Diseño y Gestión de Proyectos, Prácticas Gimnásticas y Expresivas y Prácticas Deportivas y Juegos.

El título que otorga es el de Bachiller en Educación Física, habiéndose aprobado el Marco General del Ciclo Superior de la Escuela Secundaria por Resolución n. 3828/09. Actualmente, en la provincia de Buenos Aires, son 94 los establecimientos secundarios que ofrecen la orientación (PARENTI, 2015).

\title{
La formación en Educación Física
}

En la República Argentina ejercen profesores/as de Educación Física formados en diferentes tipos de instituciones educativas del nivel superior. La carrera se encuentra radicada tanto en Universidades como en Institutos Superiores de Formación Docente (ISFD) de gestión públicas o privadas. En 2020 se dictó en 193 casas de estudio, de las cuales el 51\% eran ISFD de gestión privada, 37\% ISFD de gestión pública, $6 \%$ en Universidades públicas y $6 \%$ en Universidades privadas. Esta formación, de profesores/as, es la que habilita para el ejercicio en el sistema educativo. En concordancia con ello, la educación en los ISFD se encuentra orientada preponderantemente en dicha dirección y tiene una duración mínima de cuatro años. Además, a partir de la reforma educativa del año 1993 se implementan en 
diferentes instituciones universitarias carreras de Licenciatura de dos años que posibilitan la obtención del título universitario a los egresados del sistema terciario no universitario. Entre su primera apertura en 1993 hasta el 2020, se identificó que estas propuestas educativas se promovieron con diferentes grados de sistematicidad en treinta y tres Universidades. Además, las universidades disponen de sus carreras de licenciatura en educación física cuya duración fluctúa entre los cuatro y cinco años. Por ello, podemos encontrar profesores egresados tanto de ISFD como de Universidades, licenciados que realizaron su primera formación en ISFD y luego la completaron en las Universidades, y aquellos que la transitaron de modo íntegro en estas últimas. En la actualidad, pudimos registrar carreras de especialización y maestría en cinco instituciones. Es posible nombrar los siguientes programas de Maestría de/en: "Educación Física y Deporte" de la Universidad de Avellaneda; "Educación Corporal" y "Deporte" en la Universidad Nacional de La Plata; "Formación y Desarrollo del Rendimiento Deportivo" en la Universidad Nacional de Lomas de Zamora; "Maestría en Actividad Física y Deporte" de la Universidad de Flores; "Didáctica de la Educación Física" de la Universidad Nacional de Rosario; y, aunque actualmente no se encuentra abierta a nuevos estudiantes, la maestría en "Actividad Física y Calidad de Vida" de la Universidad Nacional de Tucumán. Hasta el momento no se pusieron en práctica programas de doctorado en Educación Física. Ahora bien, las prácticas de investigación fueron ganando visibilidad institucional tanto en términos cualitativos como cuantitativos a partir del programa incentivos a los docentes investigadores desde el año 1995 (LEVORATTI; MACARIO, 2013).

Este cuadro de situación se fue configurando a lo largo del siglo XX. Los primeros antecedentes de la formación civil en Educación Física se remontan a los "cursos temporarios de ejercicios físicos" promovidos por el médico Enrique Romero Brest desde 1901, que tenían como destinatarios a los egresados de las escuelas normales. En base a ello, en 1906 se crea el curso Normal de Educación Física, en 1909 la Escuela Normal y en 1912 el Instituto Superior en Educación Física, siempre bajo la figura del mencionado médico (SCHARAGRODSKY, 2014). Recién en 1939 abre sus puertas el Instituto Nacional de Educación Física "Gral. Belgrano" en San Fernando, Provincia de Buenos Aires. En 1953, además, se abrieron los primeros cursos en las Universidades, como fueron los casos de Tucumán y La Plata (GONZALEZ DE ÁLVAREZ, 2014; VILLA, 2003; 2011). En la década de 1960 se abrieron dos Institutos Nacionales de Educación Física (INEF), uno en Santa Fe y otro en Mendoza y durante los años 70 proliferaron los ISFD de carácter provincial, aunque bajo los planes de los INEF. En estos procesos, la Dirección Nacional de Educación Física, Deportes y Recreación y los INEF tuvieron una fuerte influencia en las propuestas formativas que se desarrollaron en el país hasta la década de 1990 (LEVORATTI, 2020). Al igual que en otros países de la región, se produjeron proyectos desarrollados por los especialistas de la Escuela de Educación Física de Colonia (Alemania), donde la formación de posgrado era uno de los ejes (DOS SANTOS, 2017; EUSSE; ALMEIDA; BRACHT, 2019). Pero, en el país no lograron concretarse en todas sus dimensiones debido a las inscripciones dentro del nivel superior y las relaciones entre los actores universitarios con los gobiernos de facto (MORAES; LEVORATTI; MARCHI JUNIOR, 2020). La carrera de Educación Física, entonces, logró su inscripción y masificación a partir de los modelos institucionales que se promovieron desde los Instituto Nacionales de Educación Física, oficiando de "modelo" para los institutos privados, los institutos provinciales y, en algunos casos, hasta en las Universidades. Ello afianzó un trayecto educativo destinado a los roles docentes. Como vimos, recién en la década de 1990 con la creación de los ciclos complementarios de Licenciatura, la apertura de los primeros proyectos de investigación en Universidades y más tardíamente los posgrados, fue que se comenzó a evidenciar otros caminos posibles para los actores de la disciplina.

En el año 2006 se sancionó la Ley Nacional de Educación n. 26.206 que, en su artículo 76, creaba el Instituto Nacional de Formación Docente (INFOD) (ARGENTINA, 2006a). Una 
de sus principales líneas de acción fue la construcción de lineamientos nacionales para las reformas de los planes de estudio de la formación docente inicial. En 2009, se publicó el texto destinado a la Educación Física bajo la denominación "Recomendaciones para la elaboración de diseños curriculares. Profesorado de Educación Física". En el mismo se consideraba que:

[...] la Educación Física, concebida como disciplina pedagógica, tiene por objeto intervenir intencional y sistemáticamente en la constitución corporal y motriz de los sujetos, colaborando en su formación integral y en la apropiación de bienes culturales específicos, a través de prácticas docentes que parten de considerarlos en la totalidad de su complejidad humana y en sus posibilidades de comprender y transformar su realidad individual y social (ARGENTINA, 2009, p. 8).

Este posicionamiento recupera debates de los enfoques críticos de la disciplina y de las ciencias de la acción motriz del portugués Manuel Sergio, preponderantemente. A partir de ello, los autores del documento buscaron distanciarse de los modelos considerados por ellos como "tradicionales" ligados al deporte y al rendimiento calificados como "elitistas", lo que exige colocar en el "centro al sujeto" de la enseñanza implicando que: "[...] los profesores sustenten su tarea pedagógica en un enfoque centrado en el sujeto en formación juntamente con los saberes que debe apropiarse para el ejercicio de su profesión, superando el logocentrismo y el magistocentrismo" (ARGENTINA, 2009, p. 66).

Estas decisiones los auto inscribió en una concepción "psicopedagógica" de la Educación Física (LEVORATTI, 2018).

Los lineamientos de la propuesta curricular estaban claramente direccionados hacia la formación de un docente, categoría que se vincula preponderantemente con el ámbito escolar. En el apartado destinado al "Campo de la formación Específica" se planteaba que: "[...] deben proponerse formar docentes de Educación Física como trabajadores de la educación para desempeñarse en diversos ámbitos; profesionales reflexivos, intelectuales críticos, autónomos y transformadores, fortaleciendo su conciencia emancipatória" (ARGENTINA, 2009, p. 42).

En base a estos lineamientos, las jurisdicciones provinciales realizaban sus diseños curriculares. De las 193 propuestas formativas que mencionamos más arriba, el 35\% se realizan en ISFD públicos o privados radicados en la provincia de Buenos Aires (LEVORATTI; SCHARAGRODSKY, 2021). El mismo año que se publicó la versión final del documento del Instituto Nacional de Formación Docente (INFoD) se sancionó un nuevo diseño curricular para los ISFD de la mencionada provincia. Este diseño fue realizado por un equipo técnico integrado por los docentes y autoridades donde se dictaba el profesorado de Educación Física y de la Dirección de Superior de la Dirección General de Cultura y Educación (LEVORATTI, 2017).

Aunque los autores del diseño, en las versiones previas colocaban el foco en la consideración de la Educación Física como una "práctica social", en la versión final del diseño se retoma la definición planteada anteriormente por el INFoD, donde el foco estaba puesto "en el sujeto". Comparando ambos documentos, el diseño provincial se singularizó por hacer referencia a la "cultura corporal" al explicitar el carácter pedagógico de la disciplina.

Con diferentes matices, en la fundamentación de los diferentes Diseños Curriculares para la Educación Física del sistema educativo provincial, se sostiene y desarrolla esta consideración de la Educación Física como disciplina pedagógica cuyas prácticas se construyen a partir de las necesidades e intereses de los sujetos, a partir de los objetos de la "cultura corporal de movimiento", pero desnaturalizados y resignificados desde el posicionamiento y el accionar crítico del docente y sus alumnos (BUENOS AIRES, 2009, p. 30). 
Además de lo planteado, la "constitución" de los "saberes específicos" se daba en vinculación con otros "campos", evidenciando un diálogo claro con el enfoque crítico propuesto por el brasileño Valter Bracht.

Al pensar los espacios de ejercicio laboral se hace referencia en el "Campo de la Práctica Docente" que durante su formación se deberá garantizar experiencias pedagógicas en: Educación Inicial, Educación Primaria, Educación Secundaria, Centros de Educación Física, como así también en "la medida de las posibilidades" en: Centros Educativos Complementarios, Gimnasios, Clubes Deportivos y Sociales, Centros de Rehabilitación, Colonias de Vacaciones, y Geriátricos.

En estas continúa apareciendo como ámbito primordial el de la docencia en los niveles educativos formales como espacios obligatorios a ser garantizados. Esto va de la mano al hecho de que las diferentes prácticas corporales que se incluían en la formación debían recuperar sistemáticamente los diseños curriculares de los niveles educativos.

Un punto que fue y es el que despertó mayor número de controversias fue la supresión de las denominaciones de los diferentes deportes y las manifestaciones gimnásticas como asignatura por la fórmula "Didácticas de las prácticas deportivas" y "Didácticas de las prácticas Gimnásticas”, respectivamente. Esto fue propuesto con el objeto de enfatizar el carácter pedagógico-didáctico que deberían tener las asignaturas por sobre el rendimiento técnico-táctico en los diferentes deportes. Las controversias se dieron porque se consideraba que a partir de ello se perdía "especificidad disciplinar" mientras que los grupos que se expresaban a favor afirmaban que la especificidad estaba en la "enseñanza" (LEVORATTI, 2017).

En lo que respecta a la problemática de género en la formación docente en Educación Física, desde la década de 1990 en los diseños curriculares se dejó de distinguir entre programas para varones y para mujeres. Ahora bien, en las asignaturas "prácticas" esta distinción perduró. Ello estaba organizado de ese modo desde los inicios de la formación de profesores de Educación Física en nuestro país, ya que, en el Instituto Nacional de Educación Física, donde se dicta por primera vez la carrera, los alumnos y las alumnas cursaban en distintos edificios, estando las clases de las estudiantes a cargo de profesores y profesoras, y las clases de los estudiantes sólo a cargo de profesores (SCHARAGRODSKY, 2006).

En este apartado se buscó presentar un panorama amplio que dé cuenta de las inscripciones institucionales de la formación en la Argentina y de los principales debates que se fueron dando en la materia, focalizando en aquellas que se dieron en el territorio de la Provincia de Buenos Aires. En base a ello, podemos afirmar que las últimas tres décadas se imprimieron cambios sustanciales en las inscripciones dentro del nivel superior de la carrera y en los lineamientos. Estos se produjeron en la urdimbre que se configuró entre los cambios en las políticas educativas, debates del campo pedagógico y los que se produjeron en la Educación Física. Sin embargo, es necesario advertir que todavía los mismos son objeto de resistencias y tensiones por parte de grupos disciplinares, aunque paulatinamente se fue consolidando una perspectiva que considera a los saberes de las Ciencias Sociales y de la Educación en sus andamiajes conceptuales.

\section{Otros aspectos de la situación de la Educación Física argentina}

En este apartado final, pretendemos presentar otros aspectos no abordados en anteriores secciones, que creemos que aportan a dar una idea general de la situación de la educación argentina en la actualidad.

En lo que respecta a competencias deportivas escolares, a nivel nacional, se organizan los "Juegos Nacionales Evita" que abarcan numerosas y variadas disciplinas deportivas en los 
que pueden participar niños/as y jóvenes de entre 10 y 18 años, así como mayores de 60 años. Además, existen juegos intercolegiales organizadas por las propias provincias (como los Juegos Bonaerenses, Juegos Intercolegiales de la Puntanidad -San Luis-, Los Juegos Intercolegiales de San Juan, entre otros) y por los municipios.

Por otro lado, en lo que refiere a la situación de la Educación Física en el país, el Centro de Implementación de Políticas Públicas para la Equidad y el Crecimiento (CIPPEC), en un informe de 2009, diagnosticó escasez y desigualdad de la actividad física en niños y jóvenes, baja intensidad de la actividad física en las escuelas, y desigualdades sociales en la práctica de la actividad física y deportiva (VELEDA et al., 2009). Además, encontraron insuficientes instalaciones para la Educación Física escolar, orientación dispar de las políticas provinciales de educación física, intervención acotada de los municipios en la gestión de la educación física. Así, por ejemplo, a partir de un informe de la UNESCO-UIS de 2008, plantea que el porcentaje de alumnos que asiste a escuelas con espacios para la actividad física en la Argentina es de 44,8\%, mientras que en países de la zona es superior: Uruguay 47\%, Brasil 53,3\%, Perú 69,8\%, Paraguay 73,0\% y Chile 80,7\% (UNESCO-UIS, 2008).

Otro de los elementos que queremos destacar se vincula con la orientación deportivista que presenta la Educación Física en Argentina, como afirman autores como Néstor Hernández y Carlos Carballo (2002) o Raúl Gómez (2009), quienes plantean que ciertas formas gimnásticas se han transformado en deporte y que el juego muchas veces se presenta como antecedente del deporte o como medio de iniciación al mismo. Además, autores como Pablo Kopelovich (2017), a partir de indagaciones empíricas han hallado una fuerte matriz deportivista en el dictado de la materia en el nivel secundario en escuelas de elite.

Con respecto a la división para las clases de Educación Física entre alumnos y alumnas, estaba avalada por una normativa vigente a lo largo de la historia, que incluyó también que los profesores enseñaran a los varones y las profesoras hicieran lo propio con las mujeres. Como cita Jorge Saraví (1995), en una circular de la Provincia de Buenos Aires de 1939 se afirma en relación a la Educación Física que "[...] las direcciones de los establecimientos determinarán los horarios de esta asignatura en forma tal que las clases sean dictadas por profesores del mismo sexo que los alumnos". Además, ese mismo año, desde el Ministerio de Justicia e Instrucción Pública se abogaba por la agrupación "por sexo para la enseñanza de la educación física" (p. 5) en las Escuelas Normales Mixtas y Colegios Nacionales del Interior, y porque la enseñanza esté a cargo de un docente también del mismo sexo (Resolución Ministerial del 15 de mayo de 1939 - ARGENTINA, 1939).

En la actualidad, al menos para la provincia de Buenos Aires, la Comunicación Conjunta n. 04/13 (Resolución n. 2476/13), establece “[...] la conformación del grupo de clase será por sección favoreciendo la grupalidad, la integración de género y la atención a la diversidad" (BUENOS AIRES, 2013, p. 9). Esa normativa está en línea con la Ley Nacional de Educación Sexual Integral (ARGENTINA, 2006b) y la Ley Nacional de Identidad de Género (ARGENTINA, 2012). Es importante destacar, también, en ese contexto, la Ley del Matrimonio Igualitario (ARGENTINA, 2010). Sin embargo, investigaciones recientes muestran el incumplimiento de la mencionada resolución (KOPELOVICH, 2017; 2019).

Por otro lado, en lo que hace al desempeño profesional de los/as profesores/as de Educación Física existe, a nivel nacional, una institución tendiente a nuclear y proteger a los/as profesionales de la Educación Física. Se trata de la "Asociación Docentes de Educación Física y Curriculares" (ADEF), que deriva de la "Asociación de Profesores de Educación Física", creada en la década de 1930, por iniciativa de Enrique Carlos Romero Brest. Asimismo, han surgido en distintas provincias argentinas iniciativas tendientes a cumplir la misma función. Por ejemplo, en La Pampa existe el "Consejo de Profesionales en Educación Física" (creado en 1982); en Salta cuentan con el "Colegio de Profesionales de Educación Física" (conformado en 2003); en Córdoba tienen la Asociación de Profesores de Educación 
Física Outdoor (APEFO); mientras que en la provincia de Buenos Aires la Unión de Profesionales de Educación Física (UPEF) lucha por la sanción de una ley para crear el "Colegio de Profesionales en Educación Física". Además, algunas de las instituciones conformadas nuclean docentes a nivel distrital, como sucede en la provincia de Buenos Aires con la Asociación de Profesores de Educación Física del Sur (Bahía Blanca) o la Asociación de Profesores de Educación Física de Mar del Plata. Finalmente, se da el caso de entidades que nuclean profesionales de la disciplina a partir de los distintos deportes, como es el caso de la Asociación de Profesores de Educación Física del Fútbol Argentino (APEFFA). Esta cuestión visibiliza las inscripciones en diferentes ámbitos laborales de los egresados en Educación Física.

\section{Consideraciones finales}

La República Argentina legisla la Educación Física escolar a partir de normativas nacionales y jurisdiccionales (provincias y Ciudad de Buenos Aires). Las primeras están dadas, principalmente, por los núcleos de aprendizajes prioritarios (NAPs), que para el nivel primario pretende el cuidado de sí mismo, de los otros y del ambiente, y para el nivel secundario se centra en la búsqueda de la disponibilidad corporal; y por el Área Nacional de Educación Física, deporte y salud, que persigue la formación de ciudadanas y ciudadanos críticos y conscientes de sus derechos y responsabilidades. En lo relativo a la legislación jurisdiccional, para el caso de la provincia de Buenos Aires, la Educación Física es concebida como práctica social que se manifiesta en diferentes actividades motrices propias de la cultura y como disciplina pedagógica en el ámbito educativo. Asimismo, en dicha provincia se ofrece, para el ciclo superior, la orientación en Educación Física, que comprende un conjunto de conocimientos y experiencias que permiten a los/as jóvenes profundizar saberes corporales y motrices en ámbitos variados, para la conquista de su disponibilidad corporal y motriz y para tener acceso a la cultura corporal de modo reflexivo, crítico y fundamentado. En ese marco, las clases de la asignatura que nos compete son obligatorias a lo largo de 13 años de escolaridad (desde el último año del nivel inicial hasta el último año de la escuela secundaria).

La formación docente en Educación Física se da en distintos formatos, desde instituciones tanto públicas (y totalmente gratuitas) como privadas, dependientes del Estado Nacional (como las Universidades) o de las distintas jurisdicciones (como los Institutos Superiores de Formación Docente). Así, se ofrecen tanto profesorados como licenciaturas. Además, en distintas partes del país se dictan especializaciones y maestrías sobre Educación Física y deportes, no existiendo hasta el momento, Doctorados sobre la disciplina. En este ámbito, desde una perspectiva de género, han sido dejadas de lado las prácticas diferenciadas por sexo, que marcaron los primeros años de su existencia, hacia inicios del siglo XX.

En lo que hace a la situación de la disciplina en Argentina, también queremos destacar que predomina cierta deportivización, lo que hace que contenidos como los juegos y las gimnasias se transformen en deporte o sean subsidiarios del mismo. Eso se ve, por ejemplo, en una serie de competencias deportivas escolares nacionales y provinciales.

Por otro lado, nos encontramos que, al menos en la provincia de Buenos Aires, desde 2009, en línea con Leyes Nacionales, se prohíbe la división de los grupos por género para las clases de Educación Física. En cambio, se favorece la grupalidad, la integración de género y la atención a la diversidad. Esta problemática no tuvo el mismo desenlace en las diferentes provincias donde todavía en algunos casos perdura la división de acuerdo al "sexo" de los estudiantes. 
Asimismo, con respecto al desempeño profesional de los/as docentes de Educación Física, existen a nivel nacional, provincial y municipal, distintas entidades que nuclean a esos/as profesionales, acompañando su labor y luchando por legislación que los/as protejan.

Para cerrar, en relación a los desafíos pendientes para la Educación Física, entendemos que queda mucho por mejorar: las condiciones edilicias y materiales en las escuelas; la inclusión en la institución escolar en términos de género, personas con discapacidad, y sujetos de pueblos originarios; la concreción de una asignatura que supere el deporte centrismo; entre otras cuestiones. Además, adherimos a la necesidad de continuar con la jerarquización de la disciplina, con la concreción de mayor reconocimiento social.

\section{Referencias}

ARGENTINA. Ministerio de la Relaciones Exteriores, Comercio Internacional y Culto. Demarcación del límite exterior de la Plataforma Continental: una política de Estado convertida en ley. Disponible en: https://cancilleria.gob.ar/es/demarcacion-del-limite-exterior-de-la-plataforma-continental-una-politica-de-estadoconvertida-en. Accedido en: 26 ene. 2022.

ARGENTINA. Ministerio de Educación. Área Nacional de Educación Física, Deporte y Salud. Disponible en: https://www.argentina.gob.ar/educacion/politicaseducativas/educacion-fisica. Accedido en: 10 sept. 2021.

ARGENTINA. Ministerio de Educación. Resolución n. 272/19, de 9 de diciembre de 2019: Marco de Organización para la enseñanza de la Educación Física escolar en Argentina. Secretaria de Gestión Educativa, 2019.

ARGENTINA. Ministerio de Justicia y Derechos Humanos de la Nación. Ley n. 26.743, de 9 de mayo de 2012 : Identidad de Género. Secretaría de Derechos Humanos, 2012.

ARGENTINA. Ministerio de Educación. Núcleo de Aprendizajes Prioritarios. Nivel Secundario. Consejo Federal de Educación, 2011.

ARGENTINA. Ministerio de Justicia y Derechos Humanos de la Nación. Ley n. 26.618, de 15 de julio de 2010 : Matrimonio Civil. Secretaría de Derechos Humanos, 2010.

ARGENTINA. Ministerio de Educación. Recomendaciones para la elaboración de diseños curriculares. Profesorado de Educación Física. Ciudad Autónoma de Buenos Aires: Ministerio de Educación, 2009.

ARGENTINA. Ley n. 26.206, de 14 de diciembre de 2006: Ley de Educación Nacional. 2006a.

ARGENTINA. Ley n. 26.150, de 4 de octubre de 2006: Programa Nacional de Educación Sexual Integral. Consejo Federal de Educación, 2006 b.

ARGENTINA. Ministerio de Educación. Núcleo de Aprendizajes Prioritarios. Nivel Inicial. Consejo Federal de Educación, 2004a.

ARGEnTINA. Ministerio de Educación. Núcleo de Aprendizajes Prioritarios. Nivel Primario. Consejo Federal de Educación, 2004b.

ARGENTINA. Ley n. 24195, de 14 de abril de 1993: Ley Federal de Educación. 1993.

ARGENTINA. Ministerio de Justicia e Instrucción Pública. Resolución Ministerial, de 15 de mayo de 1939: Disponiendo que los alumnos de las Escuelas Normales Mixtas y Colegios Nacionales del interior de la República serán agrupados por sexo para la enseñanza de la educación física. 1939

BUENOS AIRES (Provincia). Dirección General de Cultura y Educación de la Provincia de Buenos Aires. Diseño curricular para la educación primaria: primer ciclo y segundo ciclo. La Plata: DGCyE, 2018a.

BUENOS AIRES (Provincia). Dirección General de Cultura y Educación de la Provincia de Buenos Aires. Currículo de Jardines de Infantes. La Plata: DGCyE, 2018 b. 
BUENOS AIRES (Provincia). Dirección General de Cultura y Educación de la Provincia de Buenos Aires. Resolución n. 2476/13. La Plata: DGCyE, 2013.

BUENOS AIRES (Provincia). Dirección General de Cultura y Educación de la Provincia de Buenos Aires. Diseño Curricular para la Educación Secundaria. Orientación Educación Física. 4to, 5to y 6to año. La Plata: DGCyE, 2010.

BUENOS AIRES (Provincia). Dirección General de Cultura y Educación de la Provincia de Buenos Aires. Diseño Curricular del Profesorado en Educación Física. La Plata: DGCyE, 2009.

DOS SANTOS, F. C. Bola em jogo entre Brasil e República Federal da Alemanha: cientificidade e modelagem pedagógica na formação de professores na Escola de Educação Física da UFMG (1963-1982). 2017. Dissertação (Mestrado em Educação) - Faculdade de Educação, Universidade Federal de Minas Gerais, Belo Horizonte, Brasil, 2017.

EUSSE, K.; ALMEIDA, F.; BRACHT, V. "Esportivização" da educação física colombiana: a "herança" do convênio Colombo-Alemão nas páginas da revista Educación Física y Deporte. Revista Brasileira de Ciências do Esporte, v. 41, n. 4, p. 437-443, 2019.

GÓMEZ, R. Género y didáctica de la Educación Física. Subjetividad y cuerpo propio en la infancia. Revista Lecturas: Educación Física y Deportes, v. 8, n. 47, 2002.

GONZALES DE ÁLVAREZ, M. L. Instituto de educación física de la UNT primeros tiempos de la formación de profesores en la Universidad (1953/1959). Mimeo, 2014.

HERNÁNDEZ, N.; CARBALLO, C. Acerca del concepto de deporte. Alcance de su(s) significado(s). Revista de Educación Física y Ciencia, v. 6, p. 87-102, 2002.

KOPELOVICH, P. Fútbol como práctica de exaltación de las masculinidades. El caso de un colegio secundario de sectores medios de la provincia de Buenos Aires (Argentina). Revista Cuadernos, n. 55, p. 65-84, 2019.

KOPELOVICH, P. Educación Física y escuela secundaria. Sentidos y prácticas en torno a la enseñanza de la asignatura en un colegio de la provincia de Buenos Aires (Argentina). Tesis (Maestría en Ciencias Sociales con Orientación en Educación) - Facultad Latinoamericana de Ciencias Sociales, 2017.

LEVORATTI, A.; MACARIO, P. La lógica perversa y las exigencias académicas. Una aproximación a las perspectivas de los profesores en Educación Física de la Universidad Nacional de La Plata (UNLP) sobre el Programa de Incentivos a los Docentes- Investigadores. Revista Brasileira de Ciências do Esporte, v. 32, n. 2, p.341-357, 2013.

LEVORATTI, A.; SCHARAGRODSKY, P. Notas para una historia de las instituciones argentinas de formación de docentes en educación física durante el siglo xx. Revista Iberoamericana de Educación Superior (RIES), v. 12 , n. 35, p. 92-110, 2021.

LEVORATTI, A. La configuración de la Dirección Nacional de Educación Física, Deportes y Recreación de la República Argentina. Entre las problemáticas locales y los lineamientos internacionales (1963-1983). Revista Espacio Abierto. Cuaderno Venezolano de Sociología, v. 29, n. 2, p. 110-129, 2020.

LEVORATTI, A. Los debates de la educación física y los enfoques de las ciencias sociales. Un análisis de los lineamientos curriculares nacionales destinados a la formación docente en Argentina. (1993-2015). The Journal of the Latin American Socio-cultural Studies of Sport, v. 9, n. 1, p. 45-63, 2018.

LEVORATTI, A. Configuraciones de la formación de los profesores en educación física. Actores y sentidos en disputa en instituciones de educación superior en la provincia de Buenos Aires (Argentina, 1990-2015), 2017. 430 p. Tese (Doctorado en Ciencias Sociales y Humanas) - Universidad Nacional de Quilmes, Buenos Aires, 2017. 
Alejo Levoratti; Pablo Kopelovich

MORAES, L. C. L.; LEVORATTI, A.; MARCHI JÚNIOR, W. A sociologia do esporte na Argentina: apontamentos preliminares acerca da estruturação de um subcampo acadêmico-científico. Movimento, v. 26, p.e26097, 2020.

PARENTI, C. El deporte escolar en la provincia de Buenos Aires. In: LEVORATTI, A.; ZAMBAGLIONE, D. (compiladores). La recreación y el deporte social como medio de inclusión. Conceptualizaciones, reflexiones, debates. Berazategui: Engranajes de la Cultura, 2015. p. 225-237.

SARAVÍ, J. Hacia una Educación Física no sexista. Revista Educación Física y Ciencia, v. 1, n. 0, p. $32-39$ [112], 1995.

SCHARAGRODSKY, P. El discurso médico y su relación con la invención del oficio de 'educador físico': entre la heteronomia solapada y la autonomía vigilada (Argentina 1901 -1931). In: SCHARAGRODSKY, P. (compilador). Miradas Médicas sobre la cultura física en Argentina 1880-1970. Ciudad Autónoma de Buenos Aires: Prometeo Libros, 2014. p. 101-148.

UNESCO. Sistema de Información de Tendencias Educativas en América Latina. Argentina, Perfil del país. UNESCO, SITEAL, Buenos Aires, 2016.

UNESCO-UIS. A view inside primary schools. A World Education Indicators (WEI) cross-national study, Montreal: UNESCO-UIS, 2008.

VELEDA, C.; TCHINTIAN, C.; COPPO, M.; GIGLI, P. Centro de implementación de políticas públicas para la equidad y el crecimiento (CIPPEC). Políticas de promoción de la actividad física y deportiva. Opciones para los niveles provincial y municipal. Buenos Aires: Fundación CIPPEC, 2009.

VILLA, A. Currículum, educación física y formación del profesorado: el caso del profesorado de educación física de la Universidad Nacional de La Plata. Ágora para la Educación Física y el Deporte, v. 13, n. 3, p. 321340, 2011.

VILLA, A. La tradición humanista en la formación de profesores/as (Amavet y el caso del Profesorado en Educación Física. UNLP). Revista Lecturas: Educación Física y Deportes, v. 8, n. 56, 2003.

Recibido en: 12 nov. 2021. Aprobado en: 27 ene. 2022. 